\title{
Distance- and spin-resolved spectroscopy of iridium atoms on an iron bilayer
}

\author{
Johannes Schöneberg, ${ }^{1, *}$ Nuala Mai Caffrey, ${ }^{2,3}$ Paolo Ferriani, ${ }^{2}$ Stefan Heinze, ${ }^{2}$ and Richard Berndt ${ }^{1}$ \\ ${ }^{1}$ Institut für Experimentelle und Angewandte Physik, Christian-Albrechts-Universität zu Kiel, D-24098 Kiel, Germany \\ ${ }^{2}$ Institut für Theoretische Physik und Astrophysik, Christian-Albrechts-Universität zu Kiel, D-24098 Kiel, Germany \\ ${ }^{3}$ Department of Physics, Chemistry and Biology (IFM), Linköping University, SE-581 83 Linköping, Sweden
}

(Received 21 June 2016; published 13 September 2016)

\begin{abstract}
The induced spin polarization of Ir atoms on a ferromagnetic Fe double layer on W(110) has been investigated with spin-polarized scanning tunneling microscopy. An unoccupied state is observed with a spin polarization exceeding $60 \%$ that is inverted with respect to the Fe layer. This inversion is due to the tunneling gap acting as an orbital and spin filter. Distance dependent measurements show that the spin polarization remains approximately constant over the entire experimentally accessible range, from far in the tunneling regime to $1 \AA$ from the point of contact formation. This is corroborated by density functional theory calculations which show that the inversion of spin polarization occurs within $0.5 \AA$ of the adatom.
\end{abstract}

DOI: 10.1103/PhysRevB.94.115418

\section{INTRODUCTION}

The tunneling magnetoresistance (TMR) effect in magnetic multilayer junctions relies on the relative magnetization alignment of two ferromagnetic layers separated by an insulating barrier and is used in magnetic field sensors. It originates from the spin-polarized density of states (DOS) of the electrodes and depends on the applied bias voltage and the electronic structure of the insulator, as well as its thickness [1-4]. The symmetry of the majority and minority states was shown to determine their decay lengths within the barrier [5] and thus the spin-polarized tunneling current and the TMR.

The detailed dependence of the TMR effect on the width of the barrier can be difficult to assess using a typical multilayered device. This can be overcome by utilizing the spin-polarized tip of a scanning tunneling microscope (STM) as one electrode and the vacuum gap between it and the second electrode as the tunneling barrier. In doing so, the energy and distance dependence of the spin polarization can be studied.

A distance dependence of the spin polarization was previously shown to occur above Ni tips [6] and above clean ferromagnetic substrates $[7,8]$. The energy dependence of the spinpolarized current was investigated for clean ferromagnetic surfaces [9-11] as well as for molecules [12-14] and single atoms [15-19] adsorbed on such surfaces. In some cases it was reported that the sign of the spin polarization above adatoms was inverted with respect to the underlying surface $[19,20]$. Analogous to the mechanism in layered TMR devices, an orbital filtering effect of the vacuum tunneling barrier was suggested to be the source of this behavior [17,19-21]. These studies found that, for $3 d$ magnetic adatoms, minority $d$ states tend to dominate at the adatom, with a much smaller contribution from majority $s$ states. However, the $d$ states decay much faster in the vacuum than the $s$ states, with the result that the majority $s$ states contribute primarily to the conductance in the vacuum. From this, it is evident that the sign of the spin polarization will be inverted at a certain tip-adatom separation. However, the distance dependence of the spin polarization above single adatoms has not been experimentally explored to

\footnotetext{
*schoeneberg@physik.uni-kiel.de
}

date and several open questions remain. For example, Alvarado predicted that for bulk Ni tips on GaAs the largest change of the spin polarization will occur at large electrode separations [6], whereas other studies reported a rather constant rate of change of spin polarization with distance in this range [8,18,22].

Here, we experimentally investigate the energy and distance dependence of the spin polarization to determine at which electrode separation the transition to noninverted spin polarization occurs. We find the sign of the spin polarization remains constant over a wide range of experimentally accessible tip-sample distances. This would suggest that the high density of minority $d$ states, known to be present at the adatom in an energy range around the Fermi level, is not detected. Density functional theory (DFT) calculations are then used to determine which orbitals contribute to the tunneling current and to verify the proposed model of spin-dependent spatial decay of the orbitals [17,20,21]. We find that the $d$ states have decayed sufficiently at distances so close to the atom that they do not directly contribute to the tunneling current through the junction. Moreover, the transition to noninverted spin polarization is critically dependent on the electron energy.

Previous experiments mainly involved $3 d$ elements, due to their high magnetic moments. Stable magnetic moments, however, may be expected for all atoms with partially filled shells. We used the $5 d$ element Ir, in which a magnetic moment is induced by coupling to a ferromagnetic Fe bilayer on a $\mathrm{W}(110)$ surface. This surface is well studied and exhibits out-of-plane polarized magnetic domains [23]. As such, it is ideally suited to spin-polarization measurements. Ir was chosen as it is the $5 d$ counterpart to Co, which was previously studied on the same surface [19], while its high atomic number may induce effects related to an increased magnetic anisotropy [24].

\section{EXPERIMENTAL AND THEORETICAL METHODS}

Experiments were performed with a STM operating at $4.6 \mathrm{~K}$ in a vacuum of $10^{-9} \mathrm{~Pa}$. W(110) was cleaned by cycles of heating to $1500 \mathrm{~K}$ in an oxygen atmosphere of $10^{-4} \mathrm{~Pa}$ and subsequent flashing to temperatures exceeding $2200 \mathrm{~K}$. Fe films were grown at a substrate temperature of $400 \mathrm{~K}$ to enable step-flow growth of the second layer. The Fe layer exhibits 

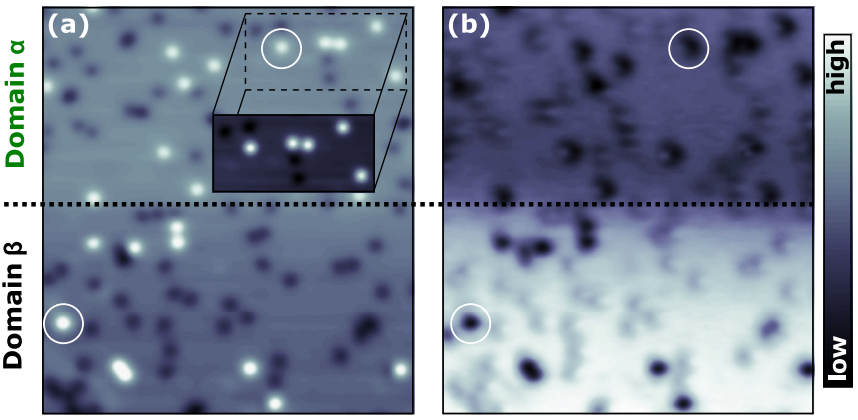

FIG. 1. (a) Topograph $\left(700 \mathrm{mV}, 32 \times 35 \mathrm{~nm}^{2}\right)$ of Ir atoms adsorbed on magnetic Fe domains measured with a spin-polarized tip. Single Ir atoms are readily distinguishable as protrusions, which is further illustrated by the inset $(1 \mathrm{~V})$ where the color scale has been adjusted. Defects of the Fe layer appear as depressions. (b) $d I / d V$ map recorded simultaneously with the topograph. At the applied sample voltage of $700 \mathrm{mV}$ the observed large scale contrast stems from differently polarized magnetic domains. Since the magnetic domains are polarized out of plane, this contrast directly reflects out-of-plane spin sensitivity of the tip. For further evaluation we refer to domains with lower (higher) $d I / d V$ signal at $700 \mathrm{mV}$ by using $\alpha$ $(\beta)$ as index. Atoms are indexed according to the domain they are adsorbed on. The positions of two Ir atoms adsorbed on differently polarized Fe domains are indicated by circles in (a) and (b).

out-of-plane polarized domains separated by domain walls of Néel type $[25,26]$. These domains are used to control the spin polarization of the adsorbed Ir adatoms, which were deposited onto the crystal at $\approx 10 \mathrm{~K}$ and appear as protrusions in constant current topographs [Fig. 1(a) and inset]. Information on the spin polarization is gained by spectroscopy of the differential conductance $(d I / d V)$ using spin-polarized tips, which were fabricated by covering paramagnetic $\mathrm{W}$ or stainless steel with Fe in situ. The existence of an out-of-plane spin polarization was verified by analyzing the contrast between the magnetic Fe domains in $d I / d V$ maps at $700 \mathrm{mV}$ [Fig. 1(b)].

DFT calculations are performed using the VASP code $[27,28]$. Structural relaxations are performed using the revised Perdew-Burke-Ernzerhof [29] parametrization of the generalized gradient approximation while the electronic structure was determined within the local spin-density approximation using the Perdew-Zunger [30] parametrization of the Ceperley-Alder data [31]. The projector-augmented wave (PAW) method [32] is used with the standard PAW potentials supplied with the VASP distribution. The plane-wave basis set is converged using a $450-\mathrm{eV}$ energy cutoff. Structural relaxations are carried out using a $17 \times 24 \times 1 k$-point Monkhorst-Pack mesh [33] to sample the three-dimensional Brillouin zone. The vacuum DOS was determined by positioning an empty sphere at the required height directly above the adatom onto which the DOS was projected. The system is modeled using a symmetric slab consisting of five atomic layers of $\mathrm{W}$ with two monolayers of $\mathrm{Fe}$ on each side. The experimental lattice constant of $\mathrm{W}$ was used $\left(\mathrm{a}_{0}=3.165 \AA\right)$. The adatom was added on each Fe surface in the hollow-site position. The minimum distance between the adatoms in adjacent unit cells is $6.33 \AA$ so that any interaction between them will be negligibly small. Additionally, a thick vacuum layer of approximately $21 \AA$ is included in the direction normal to the surface to ensure no spurious interactions between repeating slabs. The positions of the $\mathrm{Fe}$ atoms as well as the adatom are optimized until all residual forces are less than $0.01 \mathrm{eV} / \AA$.

The spin polarization $P$ is defined as

$$
P=\frac{n_{\text {maj }}-n_{\text {min }}}{n_{\text {maj }}+n_{\text {min }}}
$$

with $n_{\text {maj }}\left(n_{\min }\right)$ as the majority (minority) local DOS (LDOS). It is not possible to experimentally measure directly the LDOS, as it is coupled with the DOS of the tip, and instead we refer to the conductance asymmetry, $A$, which is defined as [34]

$$
A=\frac{(d I / d V)_{\beta}-(d I / d V)_{\alpha}}{(d I / d V)_{\beta}+(d I / d V)_{\alpha}}
$$

where $(d I / d V)_{\alpha, \beta}$ refer to $d I / d V$ spectra taken on structures (Fe domains or Ir atoms) with opposite magnetizations [see Fig. 1]. In a first approximation the spin polarization of the Fe domains or the Ir atoms, $P_{\mathrm{Fe}, \mathrm{Ir}}$, can be directly determined from the asymmetry via [9]

$$
A_{\mathrm{Fe}, \mathrm{Ir}}=P_{\mathrm{Fe}, \mathrm{Ir}} P_{\mathrm{T}} .
$$

In order to extract $P_{\mathrm{Fe}, \mathrm{Ir}}$ from the experimental data, $d I / d V$ spectra must be measured at the same tip-substrate separation on the $\alpha$ and $\beta$ domains [35]. However, the apparent heights of domains and adatoms on different domains are usually different. To refer to the same absolute height we corrected for these differences $[19,35]$. The largest challenge in interpreting the Ir spectra is the unknown spin polarization $P_{\mathrm{T}}$ of the tip, which is usually energy dependent and may be affected by tip modifications $[21,36]$. We therefore use established results from $\mathrm{Fe}$ domains [19,37], to deduce properties of the tip through a comparison of experiment and theory.

\section{RESULTS AND DISCUSSION}

Figure 2(a) shows $d I / d V$ spectra measured above oppositely magnetized $\mathrm{Fe}$ domains. Two prominent peaks at -60 and $720 \mathrm{mV}$ due to $d_{z^{2}}$ minority states $[19,37]$ are present on both domains. They are also reproduced by the calculated vacuum LDOS, $n_{\text {maj }}$ and $n_{\text {min }}$, shown in Fig. 2(b). As the peaks are observed in the $d I / d V$ signals from both domains, this implies that the tip is only partially spin polarized. Consequently, $(d I / d V)_{\alpha, \beta}$ should not directly be compared to $n_{\text {maj,min }}$. However, information about the $P_{\mathrm{T}}$ can be deduced from the vanishing of the asymmetry of the Fe domains at $V=-140$ and $630 \mathrm{mV}$ [Fig. 2(c)]. According to Eq. (3) this can only occur if $P_{\mathrm{T}}=0$ or $P_{\mathrm{Fe}}=0$. Since our calculations and previous results show that $P_{\mathrm{Fe}}<0$ [Fig. 2(f)], $P_{\mathrm{T}}$ has to vanish at these voltages. Therefore the following tip properties may be deduced: The tip is more sensitive for minority electrons for voltages below $-140 \mathrm{mV}$ (region I) and voltages exceeding $630 \mathrm{mV}$ (region III). Between these two voltages (region II) majority electrons prevail. Using this information, we can interpret the Ir data, presented in Fig. 2(d), as follows: States appear on $\beta$ atoms at 410 and $640 \mathrm{mV}$, whereas $\alpha$ atoms show a resonance at $710 \mathrm{mV}$ on a monotonously increasing background. The absence of other signatures shows that the state observed at $\beta$ atoms at $410 \mathrm{mV}$ is largely dominated by one spin channel. Because the tip is more sensitive to majority 

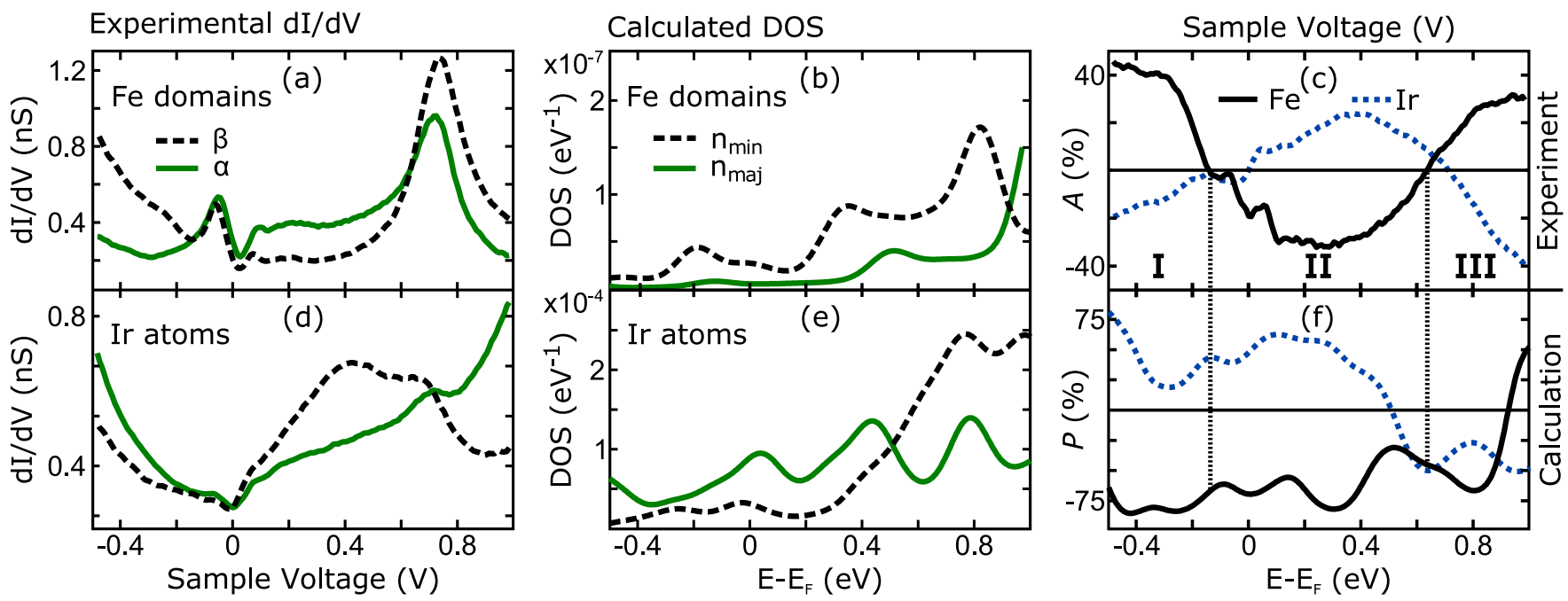

FIG. 2. (a, d) Spin-polarized $d I / d V$ spectra of Fe domains and Ir atoms, respectively. (b, e) Majority $\left(n_{\text {maj }}\right)$ and minority $\left(n_{\text {min }}\right)$ vacuum densities of states $8.6 \AA$ above the hollow site of the Fe surface and $5 \AA$ above the Ir atom, respectively. (c) Experimental asymmetries of Fe domains and Ir atoms on Fe calculated via Eq. (2) from the data in (a) and (d), respectively. (f) Corresponding calculated spin polarizations [via Eq. (1) from the data in (b) and (e)]. Comparison of (c) and (f) in combination with Eq. (3) suggests that the spin polarization of the tip changes sign between regions I, II, and III. In regions I and III the tip is more sensitive to minority states while for region II majority electrons prevail. A one-to-one correspondence between the $d I / d V$ curves and $n_{\text {maj }}$ or $n_{\min }$ may not be expected as the tip is only partially spin polarized.

electrons at this energy, we identify this peak as being the state in the majority channel at $400 \mathrm{meV}$ in the spin-polarized vacuum LDOS [Fig. 2(e)], which is predominantly of $s p_{z}$ character [cf. Fig. 3(b)]. This state is not present in the minority channel, apart from a small shoulder, in agreement with the experimental observations.

Due to the vanishing spin polarization of the tip, $A_{\mathrm{Ir}}$ vanishes at similar voltages as $A_{\mathrm{Fe}}$. Surprisingly, the signs of both asymmetries are different over almost the whole voltage range of -0.5 to $1 \mathrm{~V}$, suggesting that $\mathrm{Ir}$ is polarized oppositely to the Fe surface. This interpretation is supported by the spin polarizations calculated via the vacuum LDOS [Fig. 2(f)]. For energies between -500 and $500 \mathrm{meV}$, Ir predominantly exhibits majority states while Fe displays minority states. The vacuum spin polarization reverses sign at an energy of $500 \mathrm{meV}$ as a result of the large increase in the minority $p_{z}$ states. The agreement between experimental and theoretical results is less favorable for voltages exceeding $500 \mathrm{meV}$. We attribute the deviation to limitations of Eq. (3), which is derived assuming a vanishing bias voltage.

Close to the experimentally observed state at $410 \mathrm{mV}$ the conductance asymmetry of Ir reaches an extremal value of $25 \%$ at $370 \mathrm{mV}$. To determine the corresponding value of $P_{\mathrm{Ir}}$ the spin polarization of the tip is extracted from the $\mathrm{Fe}$ data [Figs. 2(c) and 2(f)] using Eq. (3) via $P_{\mathrm{T}}=A_{\mathrm{Fe}} / P_{\mathrm{Fe}}=$ $40 \%$. This value is similar to the previously used value of $44 \%[9,10]$, which is based on the spin polarization of $\mathrm{Fe}$ in planar junctions [38]. Consequently, the Ir spin polarization, given by $P_{\mathrm{Ir}}=A_{\mathrm{Ir}} / P_{\mathrm{T}}$, is $63 \%$. This is of the same order of magnitude as reported for Co atoms [19] and agrees well with the theoretical value [see Fig. 2(f)]. The induced magnetic moment of the $\mathrm{Ir}$ adatom is approximately $1 \mu_{B}$ ( $\mu_{B}$ : Bohr magneton) smaller than for Co (see Ref. [19]). Indeed, the calculations reveal that the hybridization between the ferromagnetic $\mathrm{Fe}$ surface and the Ir atom results in a spin polarization of the latter and an induced magnetic moment of $0.85 \mu_{B}$. This hybridization is evident in Fig. 3(a), which shows the spin-resolved total DOS of the Ir atom as well as the average total DOS of the four nearest-neighbor Fe atoms.

The filtering of the tunneling gap may be deduced by the DOS at the Ir adatom projected onto the $s p_{z}, d_{x z}+d_{y z}$, and $d_{z^{2}}$ states shown in Fig. 3(b). The majority states are dominated by the $d_{x z}+d_{y z}$ state. In particular, there is a large peak in the $d_{x z}+d_{y z}$ DOS at $-300 \mathrm{meV}$. However, this peak is not visible in the vacuum DOS [Fig. 2(e)], with the implication that this state decays quickly in the vacuum. In fact, the three peaks visible in the vacuum DOS at 50, 400, and $750 \mathrm{meV}$ correspond to majority $s p_{z}$ states at the adatom. This suggests that these states, despite their smaller DOS at the adatom, decay slowly and eventually dominate in the vacuum over the faster decaying $d_{x z}+d_{y z}$ state.

The minority DOS at the adatom, on the other hand, is dominated by $d_{z^{2}}$ states in a wide energy region around the Fermi level, with a small peak just below the Fermi level and a larger broad peak at $700 \mathrm{meV}$. The magnitude of the $s p_{z}$ states in the same energy region is considerably smaller. From this, we can conclude that minority $d_{z^{2}}$ states will provide the largest contribution to the tunneling current at distances very close to the adatom, while majority $s p_{z}$ states will prevail with increasing tip height. The asymmetry and calculated spin polarization in the vacuum $5 \AA$ above the adatom both show a positive spin polarization above the Fermi level [Figs. 2(c) and 2(f)]. Therefore, at some height smaller than $5 \AA$, the sign of the spin polarization must be inverted.

We note first that the inversion of spin polarization with tip height will also be strongly energy dependent. Consider, for instance, the energy region between -250 and $-350 \mathrm{meV}$ : the DOS at the atom is now dominated by the majority $d_{x z}+d_{y z}$ state while the positive spin polarization in the vacuum is 
(a) DOS at Ir atom and Fe layer

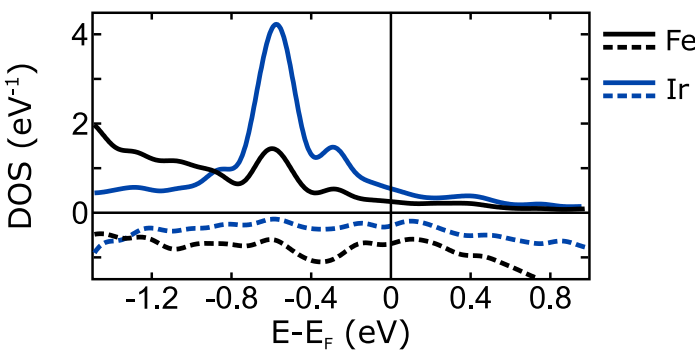

(c) Asymmetry from Experiment

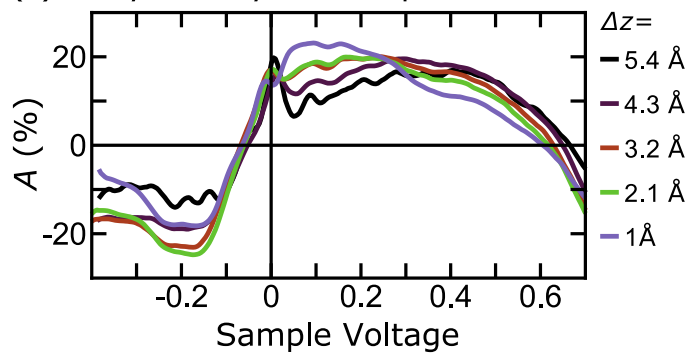

(b) projected DOS at Ir atom

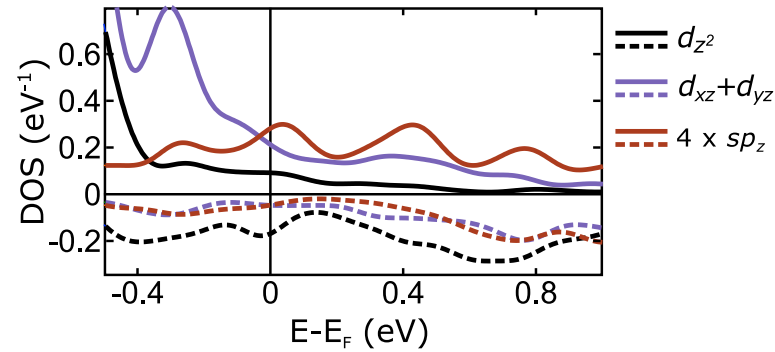

(d) Calculated spin-polarization

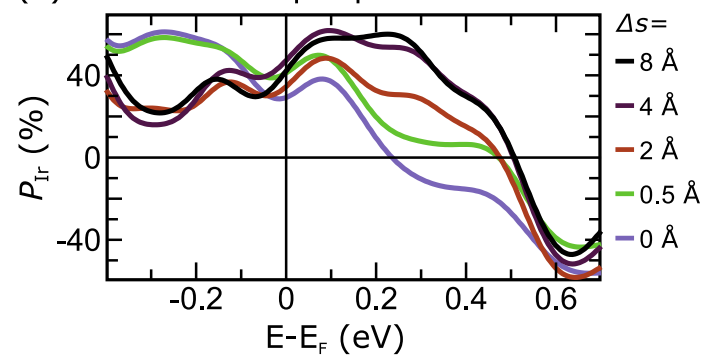

FIG. 3. (a) Total DOS of an adsorbed Ir atom and average total DOS of four nearest-neighbor Fe atoms calculated with VASP. Majority (minority) states are represented as positive (negative) values with solid (dashed) lines. (b) Orbital decomposition of the Ir DOS in (a) over a narrower energy region around the Fermi level. The $s p_{z}$ state was multiplied by 4. (c) Asymmetries of single Ir atoms measured with a spin-polarized tip at conductances from $0.4 \mathrm{nS}$ to $4 \mu \mathrm{S}$, which correspond to distances $\Delta z$ of 5.4 to $1 \AA$ from the formation of the single-atom contact at $\approx 35 \mu \mathrm{S}$. (d) Spin polarization of single Ir atoms at different distances $\Delta s$ into the vacuum calculated via DFT using VASP. $\Delta s=0 \AA$ is at the $\mathrm{Ir}$ atom.

due to the majority $s p_{z}$ state. Therefore, the sign inversion with respect to the negatively spin-polarized surface occurs directly at the adatom and so will not be observable. In contrast, for energies above the Fermi level, the sign inversion is predicted to occur at heights above the adatom due to the preponderance of negative $d_{z^{2}}$ states at the adatom. This may be expected for the state observed experimentally at $410 \mathrm{mV}$ in particular.

To further characterize the distance dependence of the filtering effect, and to determine whether an inversion of the spin polarization can be observed at any energy, we measured the asymmetry for several different tip-adatom separations. Figure 3(c) shows $A_{\text {Ir }}$ measured using the same tip at distances $\Delta z$ of 5.4 to $1 \AA$ prior to formation of the single-atom contact. $\Delta z=0 \AA$ is defined as the point of contact. Measurements in contact were not feasible due to junction instabilities at the elevated voltages required for recording $d I / d V$ curves. The asymmetry changes sign at similar voltages as before due to the varying spin polarization of the tip. Additionally, the distance dependence of $P_{\mathrm{T}}$ will also influence the magnitude of the asymmetry at different tip-adatom separations. Nonetheless, the shape of the asymmetry and thus the dominance of the majority-spin states remain stable over the entire range of $4.4 \AA$. This is in agreement with the calculated spin polarizations of Ir at different distances $\Delta s$ from the atom. The magnitude of the $P_{\text {Ir }}$ saturates at $4 \AA$ [39] [Fig. 3(d)]. It is also consistent with previous calculations of an approximately constant spin polarization for $\mathrm{Co}$ and $\mathrm{Ni}$ atomic contacts in this range [22].

The calculated spin polarization shows that the length scales involved in such inversions are indeed very small.
The spin polarization at heights of $0,0.5$, and $2 \AA$ above the adatom are also shown in Fig. 3(d). At the atom $(0 \AA) P_{\text {Ir }}$ is negative between 250 and $470 \mathrm{meV}$. By a height of $0.5 \AA$ above the atom, this is already inverted, due to the majority $s p_{z}$ state at $400 \mathrm{meV}$ [Fig. 3(b)]. The decreasing influence of the $d_{x z}+d_{y z}$ state with increasing height is particularly evident at $-300 \mathrm{meV}$. Here, it is clear that the large peak in the majority $d_{x z}+d_{y z}$ state [Fig. 3(b)] influences the spin polarization at distances very close to the adatom; a large spin polarization of $+60 \%$ is found for distances less than $1 \AA$ from the atom. By
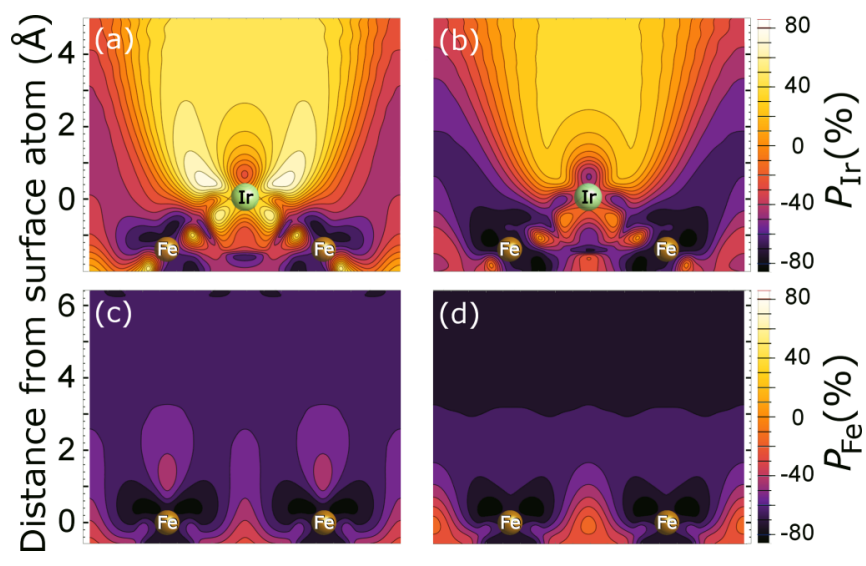

FIG. 4. Distance dependence of the spin polarization of the Ir adatom (upper panels) and the bare second Fe layer on W(110) (lower panels) integrated over the energy intervals $[-50 \mathrm{meV}, 50 \mathrm{meV}]$ (a, c) and [300 meV,400 meV] (b, d). 
$2 \AA$ from the adatom, however, the effect of this majority state is negligible. This is in agreement with previous results from single adatoms $[18,24]$, which showed that additional orbitals contribute to the conductance at contact.

Finally, Figs. 4(a) and 4(b) show slices of the spin polarization above an adsorbed Ir atom, where the spatial distribution can be seen more clearly. As a comparison, we also show the evolution of the spin polarization above a clean Fe surface in Figs. 4(c) and 4(d). For the clean surface, the spin polarization is a constant negative value over a wide range of energy and distance. In contrast, directly above the adatom the spin polarization changes sign rapidly. At the Fermi energy [Fig. 4(a)] the negative spin polarization of the clean surface is inverted directly at the Ir adatom, due to the majority $d_{x z}+d_{y z}$ state. Far from the adatom, the spin polarization remains positive, but is now carried by states with $s p_{z}$ symmetry. In contrast, at energies between 300 and $400 \mathrm{meV}$ [Fig. 4(b)], and very close to the adatom, the spin polarization is negative due to the influence of a minority $d_{z^{2}}$ state. However, with increasing height, the influence of this state wanes and the positive spin polarization associated with the $s p_{z}$ state begin to dominate with a concomitant inversion of sign. This inversion occurs at distances less than $0.5 \AA$ from the adatom, and so is not accessible with transport measurements.

\section{CONCLUSION}

In conclusion, Ir, which is paramagnetic as a bulk material, exhibits a spin polarization of similar magnitude as the well-studied $3 d$ elements, when adsorbed on an Fe surface. The tunneling current to unoccupied states exhibits a large spin polarization of more than $60 \%$, which is inverted with respect to the underlying $\mathrm{Fe}$ layer. Distance dependent measurements along with DFT calculations show that the spin polarization remains stable over a wide range of tip-adatom separations. The calculations relate this behavior to an efficient orbital filtering of the tunneling gap between tip and adatom. This filtering happens over a distance of less than $4 \AA$ from the atomic core.

\section{ACKNOWLEDGMENTS}

J.S. and R.B. acknowledge funding through SFB 668 , and N.M.C., P.F., and S.H. acknowledge funding through SFB 677 of the Deutsche Forschungsgemeinschaft. Computational facilities were provided by the North-German Supercomputing Alliance (HLRN) and the Swedish National Infrastructure for Computing (SNIC) at the PDC Centre for High Performance Computing (PDC-HPC).
[1] S. Joo, K. Y. Jung, K. I. Jun, D. S. Kim, K. H. Shin, J. K. Hong, B. C. Lee, and K. Rhie, Spin-filtering effect of thin $\mathrm{Al}_{2} \mathrm{O}_{3}$ barrier on tunneling magnetoresistance, Appl. Phys. Lett. 104, 152407 (2014).

[2] A. Kalitsov, P.-J. Zermatten, F. Bonell, G. Gaudin, S. Andrieu, C. Tiusan, M. Chshiev, and J. P. Velev, Bias dependence of tunneling magnetoresistance in magnetic tunnel junctions with asymmetric barriers, J. Phys.: Condens. Matter 25, 496005 (2013).

[3] A. Fert, A. Barthélémy, J.Ben Youssef, J.-P. Contour, V. Cros, J.M. De Teresa, A. Hamzic, J.M. George, G. Faini, J. Grollier, H. Jaffreés, H. Le Gall, F. Montaigne, F. Pailloux, and F. Petroff, Review of recent results on spin polarized tunneling and magnetic switching by spin injection, Mater. Sci. Eng. B 84, 1 (2001).

[4] G.-X. Miao, M. Münzenberg, and J. S. Moodera, Tunneling path toward spintronics, Rep. Prog. Phys. 74, 036501 (2011).

[5] W. H. Butler, X.-G. Zhang, T. C. Schulthess, and J. M. MacLaren, Spin-dependent tunneling conductance of $\mathrm{Fe} \mid \mathrm{MgO}$ | Fe sandwiches, Phys. Rev. B 63, 054416 (2001).

[6] S. F. Alvarado, Tunneling Potential Barrier Dependence of Electron Spin Polarization, Phys. Rev. Lett. 75, 513 (1995).

[7] W. Wulfhekel, H. F. Ding, and J. Kirschner, Tunneling magnetoresistance through a vacuum gap, J. Magn. Magn. Mater. 242-245, 47 (2002).

[8] M. Eltschka, B. Jäck, M. Assig, O. V. Kondrashov, M. A. Skvortsov, M. Etzkorn, C. R. Ast, and K. Kern, Probing absolute spin polarization at the nanoscale, Nano Lett. 14, 7171 (2014).

[9] M. Bode, M. Getzlaff, and R. Wiesendanger, Spin-Polarized Vacuum Tunneling into the Exchange-Split Surface State of Gd(0001), Phys. Rev. Lett. 81, 4256 (1998).
[10] S. N. Okuno, T. Kishi, and K. Tanaka, Spin-Polarized Tunneling Spectroscopy of Co(0001) Surface States, Phys. Rev. Lett. 88, 066803 (2002).

[11] O. Pietzsch, A. Kubetzka, M. Bode, and R. Wiesendanger, Spin-Polarized Scanning Tunneling Spectroscopy of Nanoscale Cobalt Islands on $\mathrm{Cu}(111)$, Phys. Rev. Lett. 92, 057202 (2004).

[12] C. Iacovita, M. V. Rastei, B. W. Heinrich, T. Brumme, J. Kortus, L. Limot, and J. P. Bucher, Visualizing the Spin of Individual Cobalt-Phthalocyanine Molecules, Phys. Rev. Lett. 101, 116602 (2008).

[13] N. Atodiresei, J. Brede, P. Lazić, V. Caciuc, G. Hoffmann, R. Wiesendanger, and S. Blügel, Design of the local spin polarization at the organic-ferromagnetic interface, Phys. Rev. Lett. 105, 066601 (2010).

[14] J. Brede and R. Wiesendanger, Spin-resolved characterization of single cobalt phthalocyanine molecules on a ferromagnetic support, Phys. Rev. B 86, 184423 (2012).

[15] Y. Yayon, V. W. Brar, L. Senapati, S. C. Erwin, and M. F. Crommie, Observing Spin Polarization of Individual Magnetic Adatoms, Phys. Rev. Lett. 99, 067202 (2007).

[16] B. W. Heinrich, C. Iacovita, M. V. Rastei, L. Limot, J. P. Bucher, P. A. Ignatiev, V. S. Stepanyuk, and P. Bruno, Spin structure of an atomic protrusion: Probing single atoms on cobalt nanoislands, Phys. Rev. B 79, 113401 (2009).

[17] D. Serrate, P. Ferriani, Y. Yoshida, S.-W. Hla, M. Menzel, K. von Bergmann, S. Heinze, A. Kubetzka, and R. Wiesendanger, Imaging and manipulating the spin direction of individual atoms, Nat. Nanotechnol. 5, 350 (2010).

[18] M. Polok, D. V. Fedorov, A. Bagrets, P. Zahn, and I. Mertig, Evaluation of conduction eigenchannels of an adatom probed by an STM tip, Phys. Rev. B 83, 245426 (2011). 
[19] N. Néel, P. Ferriani, M. Ziegler, S. Heinze, J. Kröger, and R. Berndt, Energy-resolved spin-polarized tunneling and exchange coupling of $\mathrm{Co}$ and $\mathrm{Cr}$ atoms on Fe islands on W(110), Phys. Rev. B 85, 155406 (2012).

[20] L. Zhou, F. Meier, J. Wiebe, and R. Wiesendanger, Inversion of spin polarization above individual magnetic adatoms, Phys. Rev. B 82, 012409 (2010).

[21] P. Ferriani, C. Lazo, and S. Heinze, Origin of the spin polarization of magnetic scanning tunneling microscopy tips, Phys. Rev. B 82, 054411 (2010).

[22] M. Häfner, J. K. Viljas, D. Frustaglia, F. Pauly, M. Dreher, P. Nielaba, and J. C. Cuevas, Theoretical study of the conductance of ferromagnetic atomic-sized contacts, Phys. Rev. B 77, 104409 (2008).

[23] O. Pietzsch, A. Kubetzka, M. Bode, and R. Wiesendanger, Real-Space Observation of Dipolar Antiferromagnetism in Magnetic Nanowires by Spin-Polarized Scanning Tunneling Spectroscopy, Phys. Rev. Lett. 84, 5212 (2000).

[24] J. Schöneberg, F. Otte, N. Néel, A. Weismann, Y. Mokrousov, J. Kröger, R. Berndt, and S. Heinze, Ballistic anisotropic magnetoresistance of single-atom contacts, Nano Lett. 16, 1450 (2016).

[25] M. Heide, G. Bihlmayer, and S. Blügel, Dzyaloshinskii-moriya interaction accounting for the orientation of magnetic domains in ultrathin films: Fe/W(110), Phys. Rev. B 78, 140403 (2008).

[26] S. Meckler, N. Mikuszeit, A. Pressler, E. Y. Vedmedenko, O. Pietzsch, and R. Wiesendanger, Real-Space Observation of a Right-Rotating Inhomogeneous Cycloidal Spin Spiral by Spin-Polarized Scanning Tunneling Microscopy in a Triple Axes Vector Magnet, Phys. Rev. Lett. 103, 157201 (2009).

[27] G. Kresse and J. Furthmüller, Efficient iterative schemes for ab initio total-energy calculations using a plane-wave basis set, Phys. Rev. B 54, 11169 (1996).

[28] G. Kresse and D. Joubert, From ultrasoft pseudopotentials to the projector augmented-wave method, Phys. Rev. B 59, 1758 (1999).
[29] B. Hammer, L. B. Hansen, and J. K. Nørskov, Improved adsorption energetics within density-functional theory using revised perdew-burke-ernzerhof functionals, Phys. Rev. B 59, 7413 (1999).

[30] J. P. Perdew and A. Zunger, Self-interaction correction to density-functional approximations for many-electron systems, Phys. Rev. B 23, 5048 (1981).

[31] D. M. Ceperley and B. J. Alder, Ground State of the Electron Gas by a Stochastic Method, Phys. Rev. Lett. 45, 566 (1980).

[32] P. E. Blöchl, Projector augmented-wave method, Phys. Rev. B 50, 17953 (1994).

[33] H. J. Monkhorst and J. D. Pack, Special points for Brillouin-zone integrations, Phys. Rev. B 13, 5188 (1976).

[34] R. Wiesendanger, Spin mapping at the nanoscale and atomic scale, Rev. Mod. Phys. 81, 1495 (2009).

[35] A. Kubetzka, O. Pietzsch, M. Bode, and R. Wiesendanger, Determining the spin polarization of surfaces by spin-polarized scanning tunneling spectroscopy, Appl. Phys. A 76, 873 (2003).

[36] M. Ziegler, N. Ruppelt, N. Néel, J. Kröger, and R. Berndt, Control of spin-polarized current in a scanning tunneling microscope by single-atom transfer, Appl. Phys. Lett. 96, 132505 (2010).

[37] M. Bode, S. Heinze, A. Kubetzka, O. Pietzsch, X. Nie, G. Bihlmayer, S. Blügel, and R. Wiesendanger, MagnetizationDirection-Dependent Local Electronic Structure Probed by Scanning Tunneling Spectroscopy, Phys. Rev. Lett. 89, 237205 (2002).

[38] P. M. Tedrow and R. Meservey, Spin polarization of electrons tunneling from films of $\mathrm{Fe}, \mathrm{Co}, \mathrm{Ni}$, and Gd, Phys. Rev. B 7, 318 (1973).

[39] Assuming that the single atom contact occurs at the nextneighbor distance of $\operatorname{Ir}(2.7 \AA)$, the experimental distance $\Delta z=$ $1 \AA$ corresponds to the theoretical distance $\Delta s=(1+2.7) \AA=$ $3.7 \AA$. 